\title{
Energy-efficient pulses for deep brain stimulation
}

\begin{abstract}
Energy-efficient excitation of neural tissue is investigated in this paper through the generation of optimal shape pulses. It is essential for neural stimulation to have a large percentage of the energy in the most useful frequency range. The useful range is above the frequency of the tissue-electrode interface capacitance and below the frequency of other parasitic capacitances (small capacitances of cables, connectors). One powerful observation to determine that useful range is that it centers on the minimum phase angle of the impedance. In this study we have generated stimulation signals in the form of short chirp pulses that have $95 \%$ of energy in one decade of frequency range.
\end{abstract}

\section{Introduction}

Electrical stimulation of neural tissue (deep brain stimulation) is an established method for the treatment of neural diseases. The American Food and Drug Administration (FDA) approved deep brain stimulation as a treatment for essential tremor in 1997, for Parkinson's disease in 2002 and for dystonia in 2003 [1]. For treatment, stimulation leads are placed in the brain according to the type of symptoms to be addressed.

Practical application of the method needs extremely high energy efficiency. The power of the pulses has to be exactly controlled because the tiny electrodes with high electrical impedance can degrade themselves but they can also heat up the nerve tissue, damage it and modify the neural functioning. Nowadays mostly fully implanted devices are used for neural tissue stimulation (battery-powered heart and brain pacemakers). Their functions include continuous monitoring, signal processing, decision making and stimulation when required. They should be able to operate for years. Therefore simple and extremely low-power electronics is required for the generation of stimulation pulses with optimized waveforms.

The spectra of the stimulation pulses need to be matched with the spectral properties of the complex electrode-tissue impedance $Z(j \omega)$ for the desired neural tissue stimulation effect. The energy of the stimulation signal should desirably be concentrated into a spectrum band between indicative frequencies $f_{1}$ and $f_{2}$. We should avoid generating energy with frequencies under the lower bound $f_{1}$ where the stimulation signal can poorly penetrate into the tissue due to double-layer impedance on the electrode interfaces. The frequency above $f_{2}$ should again be avoided because there are

Rauno Gordon, Mart Min, Raul Land, Thomas Johann Seebeck Department of Electronics, Tallinn University of Technology, Tallinn, Estonia, e-mail: rauno.gordon@ttu.ee

https://doi.org/10.1515/9783110558920-015 
parasitic losses due to small capacitances and inductances in the whole stimulation pathway.

\section{Objectives}

Sequences of pulses with the repetition rates from $100 \mathrm{~Hz}$ to $200 \mathrm{~Hz}$ and durations between $100 \mu$ s to $500 \mu$ s are typically used for stimulation [2]. Our aim is to find specific waveforms for the stimulating pulses that have most of the energy in the most sensitive frequency range for the neural tissue. We try to focus as much energy as possible to a narrow energy spectrum band. The center of the frequency band would be the frequency where the impedance has its maximum imaginary part - also called the peak resistance frequency $f_{p}[3,4]$. Our metric for measuring the focussed energy is the percentage of the energy that falls into one decade of the spectrum (Fig. 1). For the most promising excitation signal we also look at the spectrum of repeating pulses with various repetition rates to see if a practical usage scenario can affect the spectrum and therefore the energy efficiency of the stimulation.

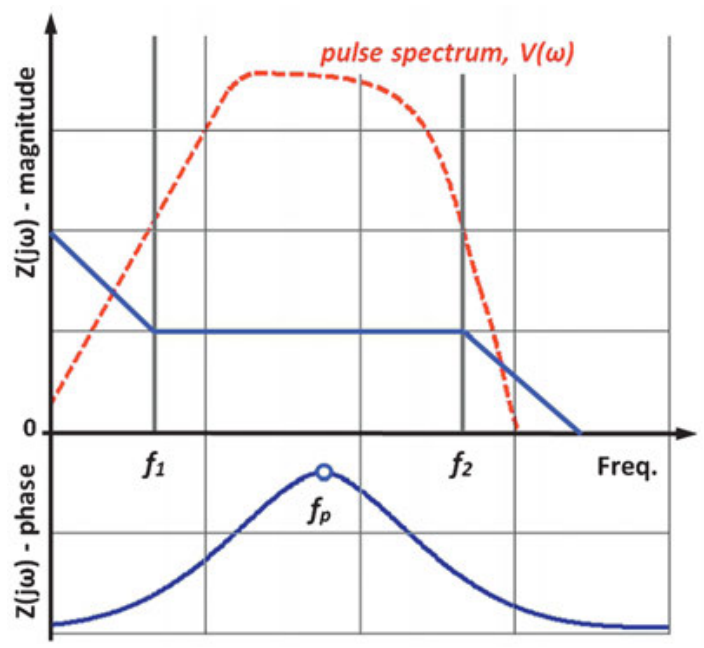

Fig. 1: Simplified magnitude and phase of tissue impedance (blue solid lines) with desired pulse spectrum (red dashed line). Frequencies $f_{1}$ and $f_{2}$ are bounds between which we want to focus the energy of the pulse; $f_{p}$ is the frequency where impedance has its minimum phase angle (peak).

\section{Methods}

Excitation pulses for nerve tissue must be exactly balanced - for a single excitation pulse there should be a positive and a corresponding negative part of the signal where electric charge given into the electrode is later balanced. If unbalanced charge is left 
into the tissue near the electrode, tissue damage can occur over time and electrode degradation can follow too, which would limit the longer-term useability of the device [5].

We have previously studied square pulses (also balanced) with and without a gap between the excitation and the balancing pulse [6]. Square pulses have the advantage of being easy to generate with simple low-power electronics. We looked into square pulses with various ratios between the amplitude and longevity of the excitation pulse and balancing pulse. We found that square pulses can be generated with up to $85 \%$ of energy in the desired band within one decade. A good and typical example is given in Fig. 2. A gap is often added in practice between the excitation and balancing part of the pulse [7]. Based on our results it did not improve the energy efficiency of the pulse but degraded it, depending on the length of the gap [6].
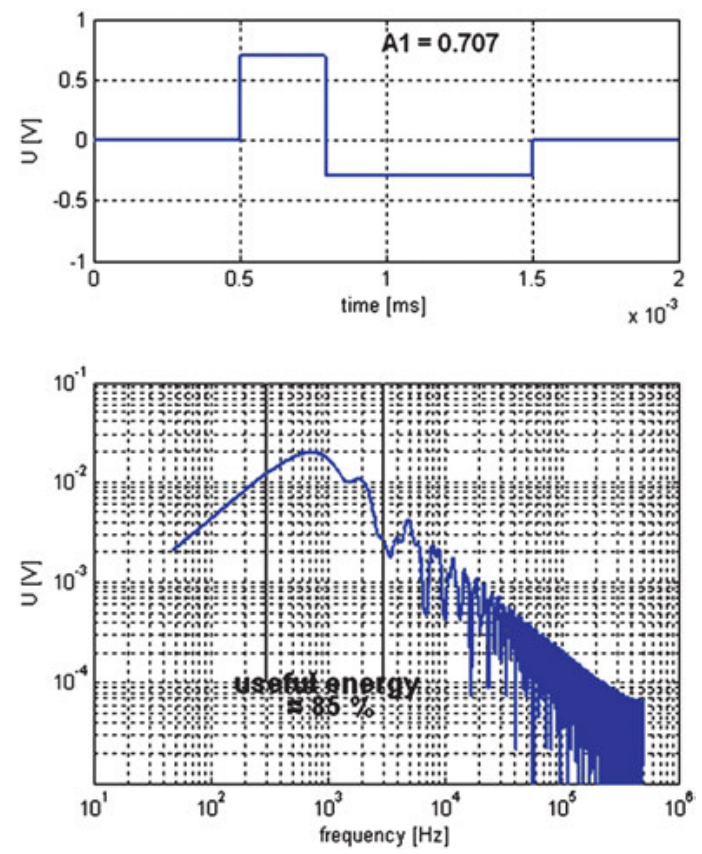

Fig. 2: Above: balanced square excitation pulse with an amplitude ratio of 0.707. Below: spectrum of the signal calculated with discrete FFT showing the bounds with gray lines where useful signal energy is calculated.

The stimulating pulses with the predetermined repetition rate and duration are synthesized mathematically for obtaining the focused and controllable energy spectrum. Several pulse signals with a frequency that rises following square function are experimented with.

Pulses of the first type are produced with frequency rising following a square function and when the sinus signal reaches the quarter-period, the frequency is lowered following the same square function. This however produces a signal that is not symmetrical with respect to the central line where the frequency rise was reversed. The 

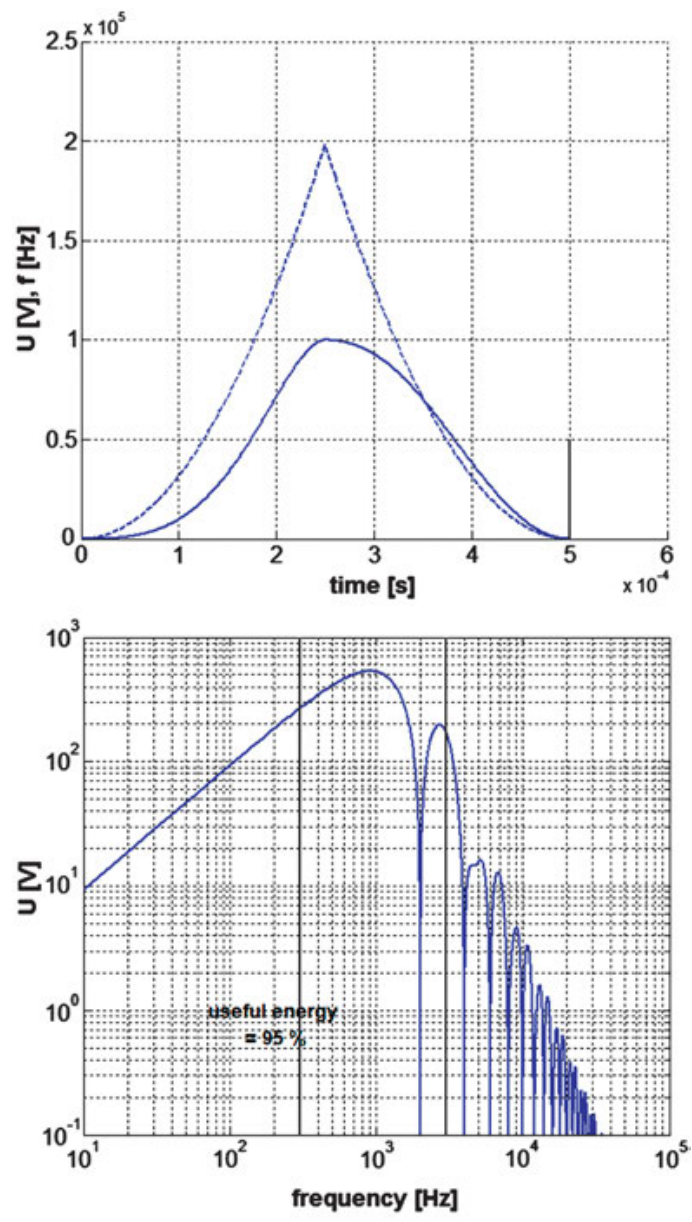

Fig. 3: Above: part of balanced signal magnitude (solid line) and rising frequency (dashed line). Below: spectrum of the signal calculated with discrete FFT shown by gray lines where the signal energy is calculated.

frequency function and an example of the pulse can be seen in Fig. 3. Please note that the pulse includes a balancing, negative part as well that is identical but not shown in the figure. After generating the balanced parts of the pulses, the frequency spectrum was calculated with discrete FFT in MATLAB. Energy within one decade of frequency was calculated for comparison purposes. The selection of the actual desired frequency band depends on the application but here the one-decade band is chosen based on typical tissue conditions.

Pulses of the second type use the rising frequency as well, but the half-period of the signal is used. The same signal in reverse sequence is then added for the balance on the negative side. The rising frequency function and the best sample pulse with both positive and negative parts is shown in Fig. 4. The spectrum and energy in one decade were calculated after that. 

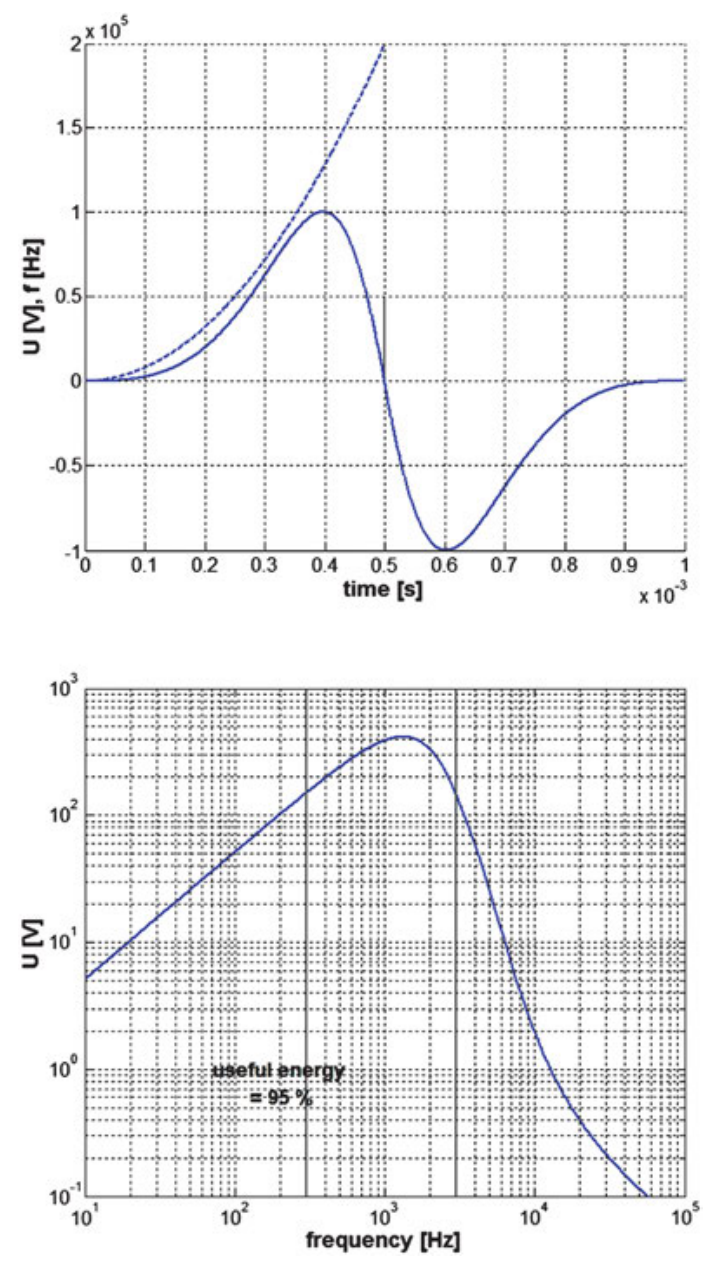

Fig. 4: Above: balanced signal magnitude (solid line) and rising frequency (dashed line). Below: spectrum of the signal calculated with discrete FFT shown by gray lines where the signal energy is calculated.

\section{Results}

The spectrum and energy efficiency of the first type of balanced signal are given in Fig. 3. We selected one decade in frequency to be the band where we desire to concentrate the energy. It can be seen that $95 \%$ of the energy resides in the desired region.

The spectrum and energy efficiency of the second type of the pulse are given in Fig. 4. The frequency spectrum exhibits a smooth and narrow curve that also concentrates around $95 \%$ of energy into the desired band.

The second type of pulse - half-cycle chirp pulse with added identical but reversed balancing part - shows a remarkable and smooth drop in energy at higher frequencies (Fig. 4). This makes it especially suitable for stimulation when parasitic losses at higher frequencies are limiting. We also investigated the spectrum of this second type of pulse in situations where the stimulation needs to be repeated. The 

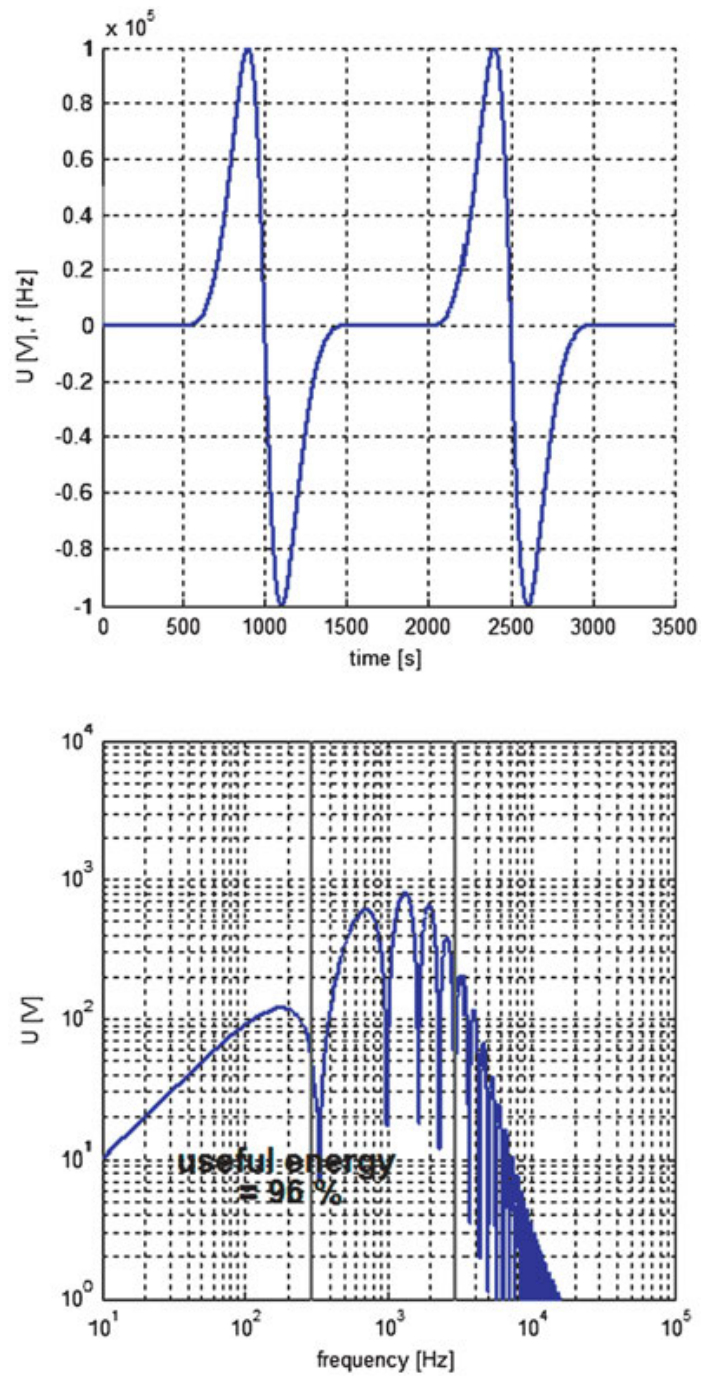

Fig. 5: Above: a signal with two balanced half-cycle chirps with a gap in between. Below: spectrum of the signal calculated with discrete FFT shown by gray lines where the signal energy is calculated. The signal retains $96 \%$ of the energy in the desired one-decade band.

results (Figs. 5 and 6) show that, even if the half-cycle balanced chirp is used for repeated stimulation, the energy efficiency is still retained above $90 \%$ in the desired one-decade band.

\section{Conclusion}

It has been found that short balanced chirp pulses (chirplets or titlets) with a duration of only a quarter or half of the full cycle concentrate about $95 \%$ of the generated 

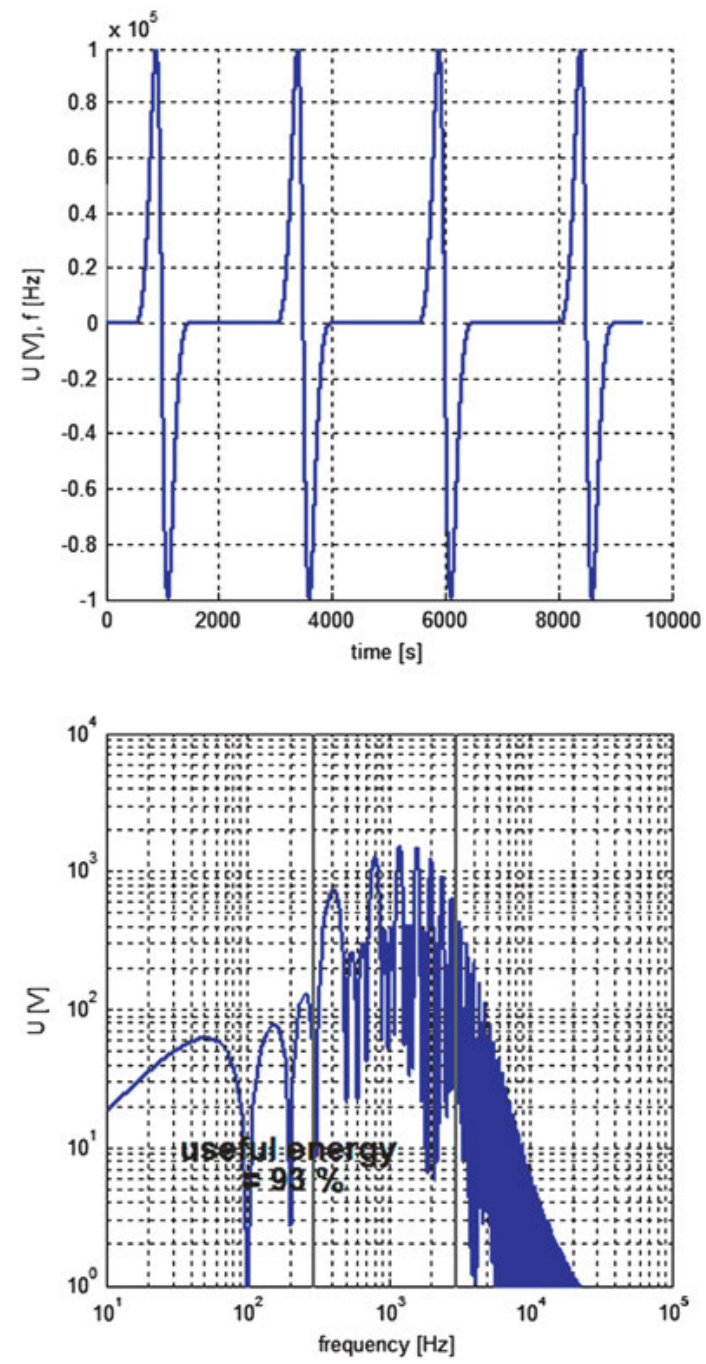

Fig. 6: Above: a signal with four balanced half-cycle chirps with a gap in between. Below: spectrum of the signal calculated with discrete FFT shown by gray lines where the signal energy is calculated. The signal retains $93 \%$ of the energy in the desired one-decade band.

energy into the most desirable frequency range from $f_{1}$ to $f_{2}$. The optimal frequency $f_{P}$ $[3,4]$ is the peak value of the phase spectrum of $Z(j \omega)$. The chirp-based stimulation pulses with optimized waveforms enable to concentrate the stimulation energy predominantly into the most sensitive frequency band. Short half-cycle chirps with an identical but reversed balancing part show remarkable concentration to the limited frequency band and are the preferred waveforms. Even if used for repeated stimulation, the spectrum stays limited to the desired band with above $90 \%$ efficiency. The true effectiveness of the balanced chirp pulses for deep brain stimulation remains to be tested in vivo. 


\section{Bibliography}

[1] A. Latteri, P. Arena, and P. Mazzone, "Characterizing deep brain stimulation effects in computationally efficient neural network models”, Nonlinear Biomedical Physics, 2011.

[2] M. K. Kuncel and M. G. Warren, "Selection of stimulus parameters for deep brain stimulation", Clinical Neurophysiology, vol. 115, 2004.

[3] United States Patent no.: 8,788,042 B2, "Apparatus and Method for Optimized Stimulation of a Neurological Target”, Mercanzini et al. Date of Patent: Jul. 22, 2014.

[4] B. Wang and J. Weiland, "Analysis of the peak resistance frequency method for extracting tissue resistance from impedance spectroscopy", Transactions on Biomedical Engineering TBME-00269, 2015.

[5] V. K. Khanna, "Neural stimulation and charge balancing approaches", In: Implantable Medical Electronics, 2016.

[6] R. Gordon, M. Min, and R. Land, "Frequency-optimized rectangular pulses for deep brain stimulation", International Journal of Bioelectromagnetism, 2015.

[7] C. R. Butson and C. C. McIntyre, "Differences among implanted pulse generator waveforms cause variations in the neural response to deep brain stimulation", Clinical Neurophysiology, vol.118, 2007. 